
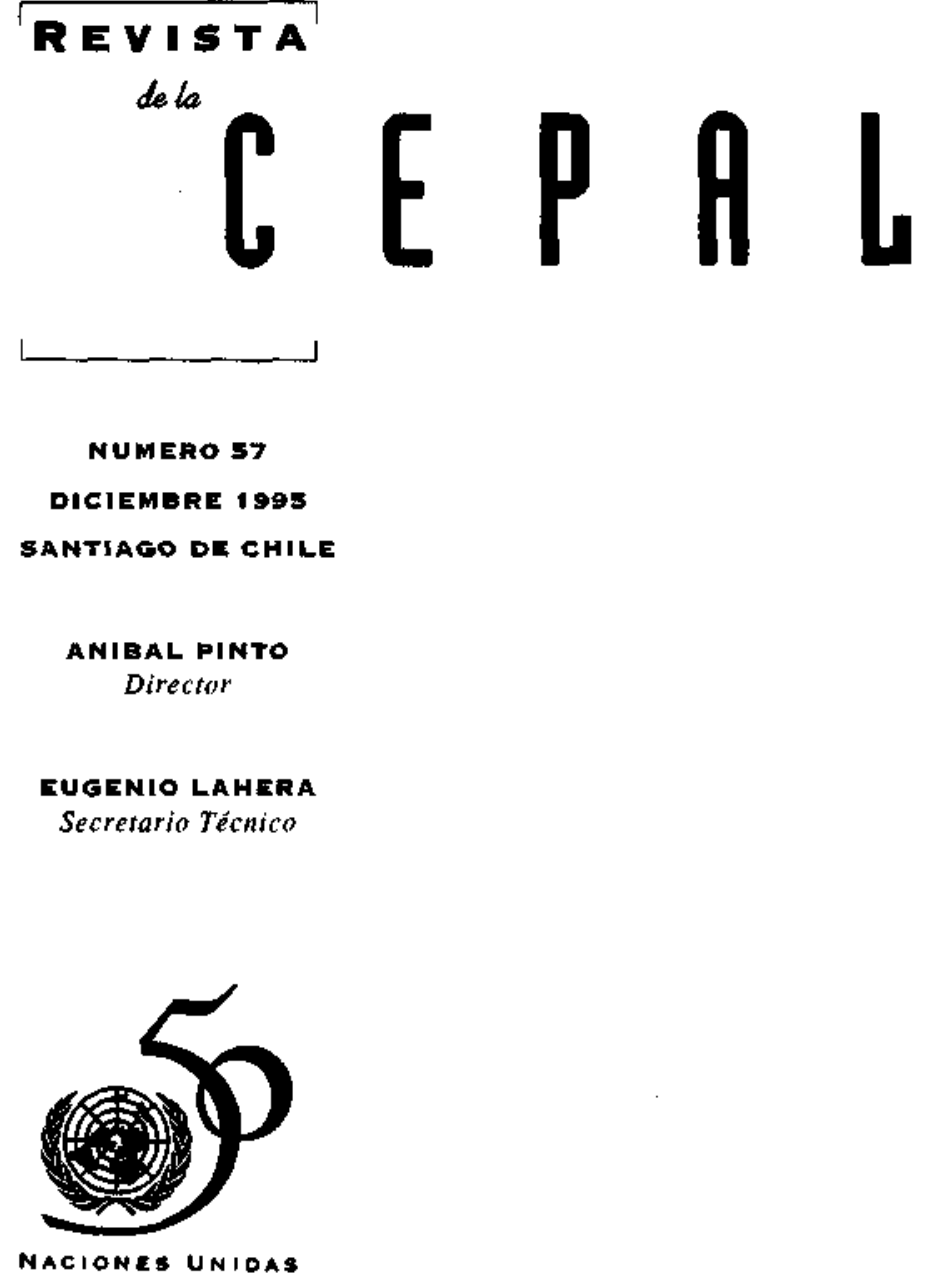
Las Naciones Unidas y la CEPAL en el

Cincuentenario de la Organización

Gert Rosenthal

La creación de las Naciones Unidas y de la CEPAL

Hernán Santa Cruz

Derechos humanos: el caso de los niños

Teresa Albánez

Gobernabilidad, competitividad e integración social

Fernando Calderón

Reforma laboral y equidad social:

la privatización de los puertos

Larry A. Burkhalter

Nuevas tendencias en las políticas salariales

Andrés E. Marinakis

Centroamérica: desempefio macroeconómico y

financiamiento social

Francisco Esquivel

Panamá y la integración económica centroamericana

Luis René Caceres

La dualidad del tipo de cambio en la economía cubana de los noventa

Archibald R.M. Ritter

Transnacionalización e integración productiva en

América Latina

Armando Di Filippo

Indice de autores y de temas en la Revista de la CEPAL, números 1 al 57

Orientaciones para los colaboradores de la Revista de la CEPAL 195 


\section{Nuevas tendencias en las políticas salariales}

\section{Andrés Marlnakis}

Organización Intemacional del Trabajo, Sección de Remuneraciones, Servicio de Derecho del Trabajo y Relaciones Laborales, Ginebra.
Hasta hace pocos años, en América Latina la política salarial era un instrumento central de la política macroeconómica, y la intervención del Estado en ella muy importante. Al comenzar los años noventa, en cambio, los países de la región se hallan, en su mayoría, en una situación macroeconómica más equilibrada y su proceso de apertura comercial se ha intensificado. Ambos factores refuerzan la importancia de los costos ante la necesidad de mantener la competitividad de la producción nacional, de modo que la búsqueda de instrumentos que flexibilicen los costos, entre ellos los laborales, es hoy una constante. Estos cambios de circunstancias y enfoque han reducido el rol del Estado en el ámbito salatial y han llevado la discusión al nivel de la empresa. Por el lado de las remuneraciones, las referencias a la flexibilidad salarial y la necesidad de ligar las remuneraciones a la productividad son permanentes, aunque por lo general ponen el acento en los supuestos beneficios macroeconómicos. En este artículo se postula que en el examen del tema no se ha dado la debida importancia a los aspectos motivacionales a nivel de la empresa y a los cambios organizativos que ella requiere, y se sugiere que los sistemas de remuneracion flexible deben adaptarse a las características de la empresa para que ésta pueda alcanzar sus objetivos estratégicos. 


\section{Introducción}

Tal como sucede en diversos ámbitos de la política económica, la política salarial está sujeta a cambios propios de su adaptación a las circunstancias económicas y al logro de ciertos objetivos. En los últimos años ha habido una serie de cambios importantes en la política salarial de algunos países latinoamericanos, que se pueden resumir en un alejamiento de las políticas de intervención en la determinación de los salarios, hacia una posición de mayor flexibilidad.

En varios países este cambio está relacionado principalmente con el proceso de ajuste y de recuperación de un cierto equilibrio macroeconómico y con el proceso de liberalización comercial, que exige una mayor competitividad internacional. Ambas situaciones tuvieron como resultado cambiar el enfoque de la política salarial, que hasta hace una década estaba fuertemente dominada por la intervención del Estado, tendiendo ahora a la determinación de los salarios a nivel de la empresa. Los temas recurrentes en este nuevo planteamiento son los costos laborales (entre los cuales los salariales son un factor importante), la productividad, la introducción de mayor flexibilidad en los costos de la empresa (siendo un componente variable del salario una de las alternativas) y los factores de motivación (entre los cuales algunas formas de remuneración pueden cumplir un papel importante).

\section{II}

\section{Objetivos generales en la}

\section{determinación de los salarios}

Los objetivos principales a los que contribuye la determinación de los salarios, juntamente con otras políticas macroeconómicas, pueden agruparse en torno a cuatro conceptos: equidad, estabilidad macroeconómica, asignación eficiente de la mano de obra y eficiencia operativa. ${ }^{1}$

En lo que hace a la equidad, hay que distinguir varias nociones. En primer lugar, la equidad está vinculada con la distribución del ingreso y el mejoramiento de la situación de los más desfavorecidos. Desde este punto de vista está íntimamente ligada al proceso de desarrollo. La equidad también está dada por la aplicación del principio de igualdad de remuneración para trabajo de igual valor. Este mismo principio implica pagos distintos para trabajos que hacen una contribución diferente a la producción, lo cual da lugar a diferencias de pago o a remuneraciones ligadas al rendimiento. Finalmente, la equidad se relaciona asimismo con la forma en que se determinan los salarios, por lo cual en muchos países se asigna un valor positivo a

\footnotetext{
1 Véase un examen más detallado de estos objetivos en olT, 1985a.
}

la negociación colectiva, que da amplias oportunidades a los empleadores y trabajadores para participar en las decisiones que atañen a sus condiciones de trabajo.

La búsqueda de la equidad está subordinada a una serie de objetivos económicos, entre los cuales destaca la estabilidad macroeconómica. Por lo tanto, la evaluación de los sistemas de determinación de salarios sopesa si ellos facilitan u obstaculizan la estabilidad de precios, la creación de empleo, el crecimiento de la producción y el equilibrio de la balanza de pagos. Tal como veremos más adelante, estas consideraciones han tenido y aún tienen un lugar preponderante en los países de América Latina.

Los sistemas de determinación salarial deberían contribuir a la asignación eficiente del trabajo, incentivando el movimiento de trabajadores desde actividades para las cuales existe exceso de oferta de mano de obra, hacia actividades para las cuales la oferta es escasa. Estos cambios deberían promover el movimiento de recursos humanos hacia sectores de mayor productividad, contribuyendo así a incrementar la eficiencia económica global. 
Finalmente, la determinación de los salarios tiene un papel esencial en la eficiencia operativa de la empresa, cual es el de maximizar la producción con un nivel dado de capital y de recursos humanos. La eficiencia en la empresa puede ser estimulada a través de la introducción de retribuciones específicas a la mejora del rendimiento de los trabajadores. Al mismo tiempo, la retribución tiene que ser percibida por los trabajadores como justa, porque de lo contrario puede dar lugar a conflictos abiertos (huelga) o encubiertos (alto ausentismo, alta rotación o baja motivación).

Es evidente que de estos cuatro objetivos el equilibrio macroeconómico es el más alejado del nivel de la empresa, mientras que la eficiencia operativa es el más cercano. Pese a esta diferencia, ambos están interrelacionados. Temas ligados a propósitos de equiljbrio macroeconómico, como la inflación o la balanza de pagos, siempre serán el marco en el que se establecerán los ajustes salariales, por ejemplo. Por otra parte, estos objetivos tienen muchas veces exigencias contrapuestas, por lo cual es preciso formular una política salarial que las contemple y que a veces lleve a soluciones intermedias. Ahora bien, si la preponderancia de un objetivo lleva a pasar por alto los restantes, es casi inevitable que se produzcan desequilibrios.

\section{III}

\section{La política salarial como instrumento de política económica}

En las últimas décadas, la política salarial ha sido uno de los instrumentos más utilizados para alcanzar el equilibrio macroeconómico en varios países de América Latina. Esto ha sucedido particularmente en los países que experimentaron altas tasas de inflación, lo cual hacía necesaria una revisión periódica de los salarios nominales. En este contexto, la intervención del Estado en el mercado de trabajo se fue haciendo más importante y compleja, manifestándose de distintas formas.

Una primera forma de intervención del Estado se dio a través de la política de fijación de salarios en el sector público, que en los países en desarrollo emplea una proporción importante de la fuerza de trabajo urbana. ${ }^{2}$ Otra fue la expansión del sector público hacia actividades productivas a través de la creación de empresas públicas, extendiendo su influencia a un sector en el que las condiciones laborales eran similares a aquellas que imperaban en el sector privado. Los salarios mínimos constituyeron también un importante instrumento de intervención; desde las primeras etapas del desarrollo industrial en América Latina, los salarios mínimos cumplieron el doble objetivo de establecer una importante regla para la inversion

\footnotetext{
2 Se calcula que entre 1975 y 1985 el empleo público total absorbía en promedio un $20 \%$ de la fuerza de trabajo no agrícola en América Latina, y alrededor de $30 \%$ en Aftica (Marinakis, 1994).
}

industrial y a la vez garantizar un ingreso mínimo a la población asalariada.

En lo que hace a la influencia de la política salarial sobre el sector privado, las experiencias son variadas, ya que la intervención del Estado ha tomado diversas formas (ort, 1991). Las negociaciones bipartitas sin intervención estatal directa representan la versión menos intervencionista. Cabe señalar que esta opción se debe apoyar en organizaciones sindicales y empresariales desarrolladas, y se puede dar en situaciones en que la negociación colectiva abarque una proporción importante de la fuerza de trabajo.

La participación del Estado en la fijación de salarios creció con su poder de arbitraje en las negociaciones colectivas. Más tarde, durante ciertos períodos en que tales negociaciones fueron suprimidas o limitadas, la función de arbitraje se transformó en la de fijación directa de normas generales y sectoriales para los reajustes salariales. En algunos casos, estos reajustes siguieron en forma directa las variadas políticas económicas. En otros casos, la introducción de algún sistema de indexación llevó a un proceso de ajustes más autónomo. Casi todos los países latinoamericanos conocieron alguna forma de intervención a través de distintos sistemas de indexación, ya fuera legalmente instituido (como en Brasil) o de aplicación informal pero extensa. 


\section{IV}

\section{Políticas salariales durante el ajuste}

En América Latina, la intervención del Estado en los distintos niveles de determinación de los salarios probablemente tuvo su apogeo en los años ochenta. Sin embargo, la eficacia de estas intervenciones se fue deteriorando a medida que la política salarial se hacía más compleja. Muchas veces, la superposición de políticas y sus múltiples objetivos llevaron a incompatibilidades en distintas áreas, por la dificultad de establecer criterios generales que satisficieran las peculiaridades y necesidades de distintos sectores.

A partir de la crisis de la deuda externa, restablecer el equilibrio macroeconómico se convirtió en el objetivo prioritario, que condicionó a los objetivos restantes y con frecuencia se contrapuso a ellos. De este modo la contención salarial se constituyó en la premisa básica del sector público y de las pautas salariales fijadas por las autoridades para el sector privado (como los salarios mínimos, las reglas de indexación o los topes a la negociación colectiva). La consecuente caída de los salarios reales ha llevado en muchos casos a una serie de desajustes, que si bien se originan en las políticas del sector público, tienen impacto sobre el mercado laboral como un todo. Aun a riesgo de caer en generalizaciones no siempre apropiadas, se puede afirmar que esta política llevo a profundos desajustes de carácter similar en muchos países. Estos desajustes se pueden agrupar en aquéllos ligados a la estructura salarial y al rendimiento, y aquéllos ligados a las remuneraciones.

\section{Desajustes Ilgados a la estructura salarial y al rendimiento}

La caída de los salarios reales en el sector público estimuló la salida de una parte del personal más productivo y capacitado, en particular en los niveles más altos. El pluriempleo se extendió y se convirtió en una práctica aceptada, aumentó el ausentismo y crecieron las prácticas ilícitas. Como forma de compensar los congelamientos salariales o la caída en los salarios reales, se aceleró el proceso de promociones, distorsionando las escalas salariales.

La intervención del gobierno a través de políticas de ingresos por lo general estuvo dirigida solamente a los salarios básicos, y con frecuencia impuso congelamientos o importantes restricciones para su ajuste. Pese a las restricciones legales, o debido a ellas, en ciertas ocasiones las empresas ampliaron otras formas de remuneración, dependiendo de su capacidad de pago. Esto tuvo dos efectos importantes. Por un lado, hizo más complejas las estructuras salariales, con lo cual en muchos casos los instrumentos utilizados no han respondido a los intereses de los trabajadores ni a los de la empresa. Por otro, al no formar parte del salario de base, tales compensaciones no han sido consideradas al determinar pensiones, por ejemplo.

Debido a la caída de la inversión en bienes de capital, se alteró el equilibrio necesario entre gastos de capital y gastos corrientes (más evidente en el caso de las empresas y servicios públicos que en la administración pública), causando grandes ineficiencias en los servicios prestados.

\section{Desajustes ligados a las remuneraciones}

Los salarios reales cayeron en todas las categorías del sector público; sin embargo, la partida presupuestaria destinada a salarios continuó siendo importante, ya que al comienzo se mantuvo el nivel de empleo.

En muchos casos se comprimío la escala salarial, ya fuese porque los incrementos otorgados a las categorías más bajas superaban a los de las categorías superiores, o bien porque correspondían a suma fija, de mayor impacto en los salarios más bajos. 


\section{Las diferencias de salarios}

Las diferencias salariales entre el sector público y el privado se incrementaron en favor de este último; las diferencias entre regiones de un mismo país crecieron cuando éstas contaban con estructuras descentralizadas, y las diferencias entre las administraciones y las empresas públicas se incrementaron en favor de estas últimas.

Hacia fines de los años ochenta, la intervención del Estado en la política salarial disminuyó apreciablemente a partir de la aplicación de ciertas políticas ligadas a los programas de ajuste. Por ejemplo, en muchos países el tamaño del Estado disminuyó debido a políticas de reducción del empleo público y, principalmente, a importantes procesos de privatización. Esto indudablemente mermó el porcentaje de la población que dependía directamente de la política salarial pública.

Un segundo ejemplo de la menor gravitación del Estado en materia salarial fue la evolución de los salarios mínimos. En la mayoría de los países latinoa- mericanos los salarios mínimos reales bajaron considerablemente durante los años ochenta. Lo que en un comienzo fue un indicador de la intención estabilizadora de las autoridades, acabó siendo una señal de flexibilización dada al mercado laboral. En muchos casos el salario mínimo perdió su significado, ya que dejó de guardar relación con algún tipo de canasta básica o con un determinado poder adquisitivo, lo cual plantea la necesidad de revisar periódicamente la forma de determinarlo.

Finalmente, la estabilización lograda por algunos países alivió la carga de ajustes salariales frecuentes y de problemas de indexación, y llevó a las autoridades a promover que los aumentos salariales estuvieran ligados a incrementos de productividad, aunque sin especificar los procedimientos para hacerlo. Este cambio de enfoque redujo el papel del Estado en el ámbito salarial y necesariamente llevó la discusión salarial al nivel de la empresa.

\section{VI}

\section{La renovada importancia de los costos laborales}

Como se dijo antes, el panorama que presentaban los países latinoamericanos en los años ochenta cambió. El comienzo de los noventa los encuentra, en su mayoría, en una situación macroeconómica más equilibrada y con índices de inflación muy bajos para la region. Este perfil diferente da una nueva dimensión a la evaluación de costos que se hace en las empresas. En el pasado los altos niveles inflacionarios, por un lado, introducían mayor imprecisión en las estimaciones y, por otro, otorgaban un mayor margen de maniobra a las empresas para transferir los mayores costos a los precios y para que los ajustes (léase caídas) de los salarios reales se dieran más por omisión que por acción. En la actualidad, en aquellos países cuyos niveles inflacionarios son bajos se necesita un mayor control de los costos de producción.

Al mismo tiempo, se ha intensificado el proceso de apertura comercial que se inició en la mayoría de los países latinoamericanos durante los años ochenta y en parte esto se debe a una tendencia internacional que promueve la liberalización. La apertura y la política cambiaria son también pilares de los planes de estabilización aplicados en varios países. Este hecho refuerza la importancia de los costos, dada la necesidad de mantener la competitividad de la producción nacional.

La renovada importancia asignada a los costos ha llevado a prestar mayor atención a los costos laborales, lo cual se expresa en dos ámbitos distintos. El primero de ellos es el de la normativa que regula aportes y contribuciones patronales, aguinaldos, pagos adicionales por vacaciones, provisiones por diversos conceptos (como indemnizaciones o accidentes de trabajo), días feriados, etc. Estos factores influirán fuertemente en la competitividad de las empresas de un país en el mercado internacional. 
Sobre la base de los costos ligados a esta normativa, un trabajo reciente compara el costo por tiempo efectivamente trabajado en relación con la remuneración neta de los trabajadores en cinco países latinoamericanos. ${ }^{3}$ En Argentina, por ejemplo, por cada 100 pesos recibidos por el trabajador la empresa abona un $103.5 \%$ adicional por día efectivamente trabajado, en Unuguay un $99.6 \%$, en Brasil un $86.5 \%$, en Paraguay un $61 \%$ y en Chile un 53.8\% (Bour, Susmel, Bagolini y Etchart, 1992).

El segundo de los ámbitos donde se expresa la relevancia de los costos laborales es a nivel de la empresa. En este caso, las posibilidades con las que se cuenta para alterar la estructura de costos están ligadas, entre otros aspectos, a su organización, estructura financiera y selección de tecnología.

Por lo tanto, la búsqueda de instrumentos que den más flexibilidad a los costos laborales son hoy una constante, tanto por parte de los gobiernos como por parte de las empresas, sobre todo en los países de mayor desarrollo de la region. Por el lado de los gobiemos, el interés de flexibilizar el mercado de trabajo radica fundamentalmente en mantener bajas las tasas de inflación y en crear empleo. Por el lado de las empresas, el objetivo es controlar los costos, ya que por ser las variaciones de sus precios menos bruscas que en el pasado (cuando las tasas de inflación eran muy altas), aun los incrementos más pequeños de los costos laborales son difícilmente compensados. Tanto para los gobiernos a partir de la normativa, como para las empresas a partir de cambios organizacionales, el objetivo común es mantener o alcanzar niveles de competitividad internacional.

Entre otros instrumentos de flexibilización, por el lado del empleo se han utilizado distintas formas de contrataciones más o menos precarias, o la subcontratación de ciertos servicios o de partes de la producción. Este tipo de flexibilidad se denomina externa o contractual. Sin embargo, esta tendencia a la precarización tiene un límite, ya que se contradice en

\footnotetext{
3 Mientras que los costos ligados a cierta normativa son mensurables en forma exacta (por ejemplo, en Brasil las contribuciones y aportes patronales son el $35 \%$ de la remuneración neta), las provisiones por concepto como accidentes del trabajo o antigüedad no son tan exactas, ya que dependen de su ocurrencia o de la estructura de los empleados de cada empresa. La estimación de estas provisiones se basa en ciertas hipótesis que introducen un margen de error. Con respecto a la antiguiedad, por ejemplo, los autores adoptaron una hipótesis de antigüedad media de 10 años. Por lo tanto. en aquellos países donde la antigiledad media es menor, el costo labotal está sobreestimado. Para mayores detalles sobre la metodología, véase Bour, Susmel, Bagolini y Etchart, 1992, pp. 6-8 y cuadros 1-5.
}

parte con la necesidad de mano de obra más calificada y polivalente que pueda satisfacer los altos niveles de calidad requeridos. Por lo tanto, otro tipo de flexibilidad se refiere a la eliminación de restricciones para la introducción de innovaciones organizacionales como el trabajo en grupo, la producción justo a tiempo y otras (Monza, 1994). Esta flexibilidad interna $o$ tecnológica es otro aspecto que refuerza la tendencia hacia una mayor flexibilidad de los sistemas de remuneración.

La importancia del tema de los costos laborales también ha variado por la serie de modificaciones que están teniendo lugar en la producción y en el sistema de remuneraciones. En primer lugar, sucesivos cambios tecnológicos y de organización productiva parecen haber reducido la gravitación relativa de los costos laborales, ya que en varios sectores ha bajado su participación en los costos totales de producción. En segundo lugar, la composición de los costos laborales ha estado cambiando porque las empresas han tendido a ampliar el uso de elementos de remuneración indirectos. En tercer lugar, se considera que la combinación de un salario básico y un componente de remuneración variable puede ser un instrumento válido para introducir flexibilidad en el sistema de pago. Y, por último, esta misma combinación abre la posibilidad de incorporar elementos motivacionales a la remuneración y de mejorar su atractivo para contratar y retener empleados calificados.

En lo que hace a la importancia de los costos laborales dentro de los costos totales, los cambios en los modos de produccion han reducido el porcentaje relativamente importante correspondiente al trabajo (especialmente al menos calificado). Este efecto es más notorio en los países industrializados; por ejemplo, en la industria japonesa de productos electrónicos el componente laboral es de 5 a $10 \%$ de los costos totales. Pero además las modificaciones no parecen estar restringidas a áreas de alta tecnología. Los cambios en la estructura de costos basados en la aplicación de nuevas tecnologías también han afectado a industrias que tradicionalmente hacen uso intensivo de mano de obra, como las textiles, lo cual en algunos casos ha revertido la ventaja comparativa en favor de los países industrializados. Se puede citar el ejemplo de Benetton, en la industria del vestuario: contrariamente a sus rivales, que han transferido su producción al Asia, esta firma fabrica un $80 \%$ de su producción en Europa (The Economist, 1994); el cambio tecnológico ha permitido a Benetton transformar la estructura de su producción 
para hacer uso intensivo de capital, con lo cual pudo mantener sus operaciones en una región de costos laborales más altos.

El peso de los costos adicionales al salario básico, como distintos tipos de bonificaciones, horas extraordinarias y otros, difiere entre sectores y entre firmas. En general se puede afirmar que la importancia de estos elementos en los costos laborales ha au- mentado por diversas razones. En las últimas décadas, el componente de los costos laborales que más creció en los países industrializados fue el de las contribuciones a los sistemas de seguridad social pagadas por los empleadores, como resultado de la mejora de esos sistemas y del mayor costo de las pensiones y la atención médica, por ejemplo (aunque recientemente éste se ha estabilizado).

\section{VII}

\section{Sistema de remuneraciones y flexibilidad}

La nueva preocupación por flexibilizar el sistema de remuneración ha contribuido a la adopción de formas indirectas de pago y de una porción variable en las remuneraciones. Desde la perspectiva de las empresas, y cualquiera sea su desempeño, lo que suele buscarse es evitar sistemas de pagos fijos a sus trabajadores. Cuando los salarios constituyen un costo fijo, en épocas de resultados negativos la dirección tiene que recurrir a despidos para bajar sus costos, lo que es siempre un proceso traumático. La introducción de un componente variable permite ajustes endogenos que reducen en forma automática los costos laborales durante los períodos de crisis y de este modo convierten a los despidos en un último recurso.

Si bien los aspectos ligados a los costos son importantes, en el debate sobre la flexibilización de las remuneraciones que se da en América Latina no se ha prestado la debida atención a aquellos aspectos motivacionales y a los cambios organizativos que se desea impulsar y que se necesitan. La introducción de un componente variable tiene como objetivo fundamental ligar la remuneración al desempeño del trabajador o de la empresa en forma inmediata y directa, para que constituya un incentivo. Pero además los incentivos materiales ligados al rendimiento deben estar orientados a mejorar el desempeño de la organización en cuestiones que le son propias. Los diversos aspectos que se debe tomar en consideración al diseñar estos sistemas deben ser adaptados a las características de la empresa, de forma tal que ésta pueda alcanzar sus objetivos estratégicos. La identificación de tales aspectos es, por lo tanto, un factor fundamental para tener éxito en la aplicación de un sistema determinado.

Los sistemas de remuneración que incluyen un componente variable pueden presentar característi- cas muy variadas en relación con diversos aspectos (Belcher, 1991):

i) La unidad cuyo desempeño va a ser medido y remunerado puede ir desde el trabajador individual, un grupo, una sección o una fábrica, hasta la empresa en su conjunto; la elección dependerá no sólo de cuestiones prácticas (dificultad para determinar la productividad de ciertos trabajadores, por ejemplo), sino también de la actitud que se quiera promover (trabajo en equipo $o$ individual).

ii) La naturaleza de lo que se remunera (como la producción, la calidad, la utilización de la maquinaria, la productividad, o una combinación de estos factores) puede variar; se debe cuidar que el incentivo creado no repercuta negativamente en otros aspectos (por ejemplo, hay incentivos para elevar la productividad que pueden llevar, en forma indeseada, a que se deteriore la calidad del producto final o aumenten los accidentes de trabajo).

iii) El nivel a partir del cual se busca mejorar el desempeño se puede fijar a través de mediciones técnicas o a partir de niveles alcanzados en el pasado.

iv) La porción salarial variable esperada debe determinarse, y ser suficiente para constituir un incentivo, pero no tan grande como para que el pago fijo caiga muy por debajo del salario de mercado. ${ }^{4}$

v) Finalmente, será preciso elegir los períodos en que los resultados serán medidos y remunerados, evitando que su frecuencia convierta su implementa-

\footnotetext{
4 Cuando se dan las condiciones para que la aplicacion de estos sistemas sea exitosa, la parte variable esperada representa entre un 25 y un $30 \%$ de la remuneración total. Si estas características no están presentes, la proporción del componente variable será menor (OIT, 1985b).
} 
ción en un costo importante y que plazos muy extensos hagan perder la identificación entre la mayor dedicación al trabajo y la remuneración adicional.

Los partidarios de incorporar un componente variable en el salario sostienen que esta alternativa ofrece, a nivel macroeconómico, beneficios en materia de empleo e inflación. Tal posibilidad hace particularmente atractivos estos sistemas para los gobiernos. En lo que hace al empleo, ligar una parte importante de la remuneración al desempeño de la empresa puede estimular nuevas contrataciones, ya que reduce el riesgo empresarial al introducir una parte variable. En lo que hace a la inflación, al vincular los aumentos de salarios a las ganancias de la empresa $o$ al incremento de la productividad se limitan las causas salariales de la inflación. Para los trabajadores, el beneficio principal de estos sistemas es que pueden aminorar los despidos cuando el desempeño de la empresa es malo, ya que la reducción del costo laboral es endógena. En épocas de crecimiento, por el contrario, los trabajadores participarán de las mejoras en forma automática.

Pese a las ventajas que estos sistemas parecen ofrecer, en la práctica tienen algunas limitaciones. A nivel macroeconómico, la limitación fundamental es que estos sistemas han sido adoptados por un número pequeño de empresas, por lo cual su impacto no es significativo. Por otra parte, al tener que centrarse en las características y objetivos específicos de las em- presas, los sistemas de remuneración variable constituyen fundamentalmente un instrumento microeconómico.

Los problemas encontrados a este nivel son muy variados. Nos podemos referir al caso de Gran Bretaña, donde se impulsó desde el gobierno, a través de exenciones impositivas, la adopción de sistemas de remuneraciones que incluyeran una parte ligada a las ganancias de las empresas. Allí se ha observado lo siguiente:

i) el componente variable por lo general se asocia a una bonificación por sobre el salario básico, y termina siendo utilizado en reemplazo del incremento salarial anual;

ii) cuando la parte variable no es un elemento significativo de la remuneración, no alcanza para compensar los altos costos en épocas de recesión y por lo tanto no evita los despidos;

iii) la empresa puede tener dificultades para retener sus mejores trabajadores durante los períodos de depresión, en los cuales el no pago de la parte variable puede hacer caer los salarios por debajo de los del mercado; y

iv) el factor elegido para determinar el componente variable puede estar expuesto a múltiples influencias que se hallan fuera del control de los trabajadores, quebrando el vínculo entre la remuneración y la dedicación al trabajo (IDS, 1990 y 1992).

\section{VIII}

\section{Aspectos cualitativos y de motivación}

Todas las consideraciones expuestas más arriba están ligadas al aspecto cualitativo del trabajo, al cual debería contribuir el sistema de remuneraciones. Dentro de estos aspectos, la política de remuneraciones de una empresa debe atraer la cantidad y calidad necesaria de trabajadores, retener esa fuerza de trabajo y contribuir a su motivación para mejorar el desempeño de la empresa. En lo que hace a la motivación, cabe destacar ante todo que la política salarial es sólo uno de los instrumentos disponibles para motivar a los trabajadores, pero que en modo alguno puede sustituir el papel que cumplen otras políticas de personal.

Los sistemas de remuneraciones que incluyen un componente variable pueden contribuir a elevar la motivación del personal y por lo general persiguen este objetivo. Entre los argumentos a favor se menciona que esos sistemas ayudan a que los empleados puedan identificarse más con la empresa e interesarse por su éxito; que además permiten estimular distintos comportamientos participativos para cambiar antiguas formas de producción, como el trabajo en equipos, la mejora de la calidad o propuestas específicas que produzcan ahorro de materiales o reducción de costos. Estos sistemas requieren una comunicación fluida entre el personal y la dirección, tanto en su diseño como en su aplicación. La falta de transparencia en esa comunicación puede llevar al fracaso del programa, y la existencia de grandes fluctuaciones debidas a factores externos fuera del control del personal puede llevar a eventuales disputas. 
Por último, cabe destacar que en el marco de la actual corriente de apertura comercial la calidad del trabajo ocupa un lugar principal, aunque no siempre reconocido. A nivel internacional los costos laborales relativos son un factor de importancia, no en forma aislada, sino en el contexto de la productividad local, la infraestructura existente, la política de gobierno, y los niveles de educación y capacitación de los trabajadores. Los costos laborales bajos per se sólo pueden ser un factor de ventaja comparativa de corto plazo. Para lograr competitividad de largo plazo, los bajos costos deben ir unidos a alta calidad. Aquellos países en los cuales tanto el costo como la calidad del trabajo sean bajos, serán más vulnerables en el nuevo contexto económico internacional.

\section{IX}

\section{Conclusiones: la necesidad de}

\section{un nuevo equilibrio}

En la primera parte de este artículo se señaló que la intervención excesiva que privilegie un objetivo y olvide los demás puede desvirtuar un sistema de remuneraciones. En el caso de América Latina graficamos este fenómeno en un período de contención salarial, con sus efectos sobre la estructura y rendimiento del empleo en el sector público en particular, y sobre las diferencias de salarios. La práctica demostró las limitaciones de varios de los instrumentos utilizados y el paso del tiempo desgastó algunos mecanismos que pudieron ser útiles en períodos más cortos. Como ejemplo de los primeros mencionaremos los intentos de aplicar una indexación parcial o límites a los incrementos salariales negociados; como ejemplo de los últimos seŕalaremos las políticas de ingresos utilizadas durante la aplicación de programas de estabilización heterodoxos.

En la actualidad, se tiende a una mayor flexibilización del mercado de trabajo en general y a nivel de la empresa, muchas veces como resultado de cambios tecnológicos $\mathbf{u}$ operativos. La adopción de algunas medidas flexibilizadoras será positiva en determinadas circunstancias, pero no se debe pensar que ésta es la única vía de solución, ni cifrar expectativas desmedidas en su capacidad de resolver problemas muy complejos.

La introducción formal de medidas flexibilizadoras no es la única alternativa, ya que incluso los sistemas tildados de rígidos pueden dar muestras de flexibilidad cuando es necesario. Un caso ilustrativo es el de Alemania, cuyo sistema de remuneraciones es frecuentemente criticado por su rigidez. Sin embargo, los sindicatos alemanes acordaron ajustes por debajo de la inflación a principios de los años ochenta, en 1989 y en 1993-1994. Además, los convenios por industria se complementan con provisiones a nivel de la empresa, lo cual da la posibilidad de limitar el pago total. En varias industrias las empresas pagan hasta un $30 \%$ por encima del mínimo establecido por convenio. Cuando las empresas quieren reducir su costo salarial, recortan ese pago adicional manteniendo el pago total constante (como en el caso de Bosch); o mantienen fijo el pago adicional, reduciendo su importancia porcentual sobre el total (como en el caso de Mercedes Benz). Otro ejemplo de flexibilidad está dado por el reciente acuerdo de Volkswagen, según el cual se recortaron las horas de trabajo, reduciendo también las remuneraciones (UMM, 1994).

Las formas salariales flexibles tienen limitaciones para alcanzar los múltiples objetivos que se les han asignado. Es importante señalarlo porque tales limitaciones afectan a los objetivos trazados tanto por los gobiemos como por los empresarios y los trabajadores.

En lo que hace a los objetivos del gobierno, no se debe esperar que los sistemas de remuneraciones variables resuelvan los problemas de empleo y que controlen la inflación. Sería más realista considerarlos una contribución, entre otras necesarias, para dar alivio relativo en situaciones de crisis a nivel de la empresa. Los sistemas de remuneración flexible deben ser vistos fundamentalmente como un instrumento útil a nivel microeconómico, pero con efectos marginales a nivel macroeconómico.

Por el lado de las empresas, este instrumento debe ser utilizado con cautela, definiendo claramente los aspectos estratégicos que se desea incentivar e 
identificando los efectos negativos que puedan surgir como resultado de su aplicación. Por otra parte, y como ya se mencionó respecto del caso de Gran Bretaña, las empresas que establecen una parte del salario en forma variable lo hacen en una proporción limitada, lo cual puede no dar el margen de flexibilidad requerido por la empresa en situaciones de caída de la demanda.

Para los trabajadores, el atractivo más evidente es el de poder participar en cierta forma de los beneficios de la empresa en los momentos de crecimiento (participación en las ganancias) o acrecentar sus ingresos en función de su mayor esfuerzo (remuneración por rendimiento), así como lograr relaciones contractuales más estables. Esto es especialmente cierto en aquellas empresas comprometidas a alcanzar mayor productividad y calidad para competir internacionalmente; ellas deberán invertir en su capital humano y estarán interesadas en reducir la rotación de sus trabajadores. En períodos de crisis, sin embargo, estas condiciones pueden sufrir cambios.

En general, los cambios flexibilizadores exigen modificaciones de comportamientos y de gestión. Las formas de remuneración ligadas, entre otras cosas, al rendimiento, la productividad y los resultados de la empresa, deben complementarse con estructuras de gestión más abiertas y participativas que hagan aceptable su aplicación, y que involucren a los trabajadores en la búsqueda de mejoras. Se ha visto que el éxito de estos sistemas depende en gran medida de la forma en que sean implantados (con la participación o no del personal) y en que se lleven adelante (formas de comunicación y seguimiento de los resultados de la empresa). Evidentemente, esto exige formas de negociación más transparentes y responsables que en el pasado, cuando las tasas de inflación altas y variables llevaban a ajustes frecuentes pero de efecto real incierto.

\section{Bibliografta}

Belcher, J. (1991): Gain Sharing, Houston, Texas, Gulf Publishing Company.

Bour, J., N. Susmel, C. Bagolini y M. Etchart (1992): Costos laborales en el Mercosur: Comparacion de los costos laborales directos. Documento de trabajo, $\mathrm{N}^{\mathrm{c}} 35$. Buenos Aires, Fundación de Investigaciones Económicas Latinoamericanas (FIEL).

IDS (Income Data Service) (1990): Profit-Related Pay, IDS Study, $N^{\circ} 471$, Londres.

(1992): Profit-Related Pay, IDS Study, № 520, Londres.

Marinakis, A. (1994): Public sector employment in developing countries: An overview of past and present trends, The Interna. tional Journal of Public Sector Management, vol. 7, $\mathrm{N}^{\circ} 2$, Bradford.
Monza, A. (1994): Flexibilidad laboral, Colección temas jurídicos, Buenos Aires, Fundación Omega Seguros, agosto.

olT (Organización Internacional del Trabajo) (1991): Employment Policies in the Economic Restructuring of Latin America and the Caribbean, trabajo presentado al Tripartite Symposium on Structural Adjustment, Employment and Training in Latin America, Caracas, agosto, Ginebra.

(1985a): Government wage policies in developing countries: Concepts and issues, Ginebra, mimeo. (1985b): La remuneración por rendimiento, Ginebra. The Economist (1994): Benetton. The next era, vol. 331, N ${ }^{\circ} 7860$, 23 al 29 de abril, Londres.

UIMM (Union des Industries Métallurgiques et Minières) (1994): Accord Volkswagen, Social Intemational. $\mathrm{N}^{\circ}$ 524, París. 